\title{
Synthetic Nonlinear RR Lyrae Instability Strips
}

\author{
R. Szabó, Z. Csubry, Z. Kolláth \\ Konkoly Observatory, Budapest, H-1121, Konkoly-Thege M. u. 13-17., \\ Hungary
}

\section{J. R. Buchler}

Physics Department, University of Florida, Gainesville, FL 32611, USA

\begin{abstract}
A recently recognized problem is revisited, namely the discrepancy between the structure of the theoretical and the empirical, observationally derived RR Lyrae instability strips. The essence of the discrepancy is that the slope of the empirical fundamental-mode blue edge is much less steep than its theoretical counterpart. The issue was investigated by means of stellar evolution and mode selection captured by nonlinear models.
\end{abstract}

\section{Introduction}

Both radiative and convective models have failed to explain the steep slope of the observed blue edges of the RR Lyrae fundamental mode $(\mathrm{F})$ and first overtone (O1) instability strips (Kolláth et al., 2000). The empirical data are coming from the extraction of the absolute magnitudes, colors, effective temperatures and metallicities directly from the observed RRab periods and light curves (Kovács \& Jurcsik, 1996, 1997; Jurcsik, 1998). Because the issue is connected to extensively debated astrophysical topics (distance scales, convection), it is very important to resolve the problem. Therefore we investigate here the effects of modal selection and evolutionary changes of physical parameters on theoretical RR Lyrae instability strips.

\section{The method}

Amplitude equations predict either-or-regions (EORs) on the HR diagram (Buchler \& Kovács, 1986) verified by hydrodynamical calculations (Kolláth \& Buchler, 2001): A star evolving from low to high $T_{\text {eff }}$ is seen as a fundamental (F) pulsator. Evolution in the opposite direction with the same stellar parameters results in an $\mathrm{O} 1$ pulsator. A similar EOR exists for an F/DM (double-mode) transition. As stars with different $\mathrm{Z}$ evolve through this region at different luminosities, theoretically a mixed area containing both RRab and RRc stars is expected, and thus modal selection might change the average slope. A nonlinear hydrocode including turbulent convection (Kolláth et al., 2002) was used to follow the modal behavior. Nonlinear edges of the possible pulsational 
modes were determined by fitting amplitude equations to series of models computed with different $T_{\text {eff }}$ at fixed $\mathrm{L}, \mathrm{M}$ and $\mathrm{Z}$. The application of advanced tools of time-frequency analysis was essential to derive instantaneous amplitudes and frequencies (Kolláth \& Buchler, 2001). Recent theoretical horizontal branch evolutionary tracks (Demarque et al., 2000) were chosen and interpolated in time. Synthetic HRDs were constructed by generating a sample of horizontal branch stars. The pulsational state(s) corresponding to every point on the evolutionary track was determined by taking into account the EORs' interpolated widths and the direction of evolution. Outside the EORs every point was considered as $\mathrm{F}, \mathrm{O} 1$ or DM pulsator depending on its $T_{\text {eff }}$ value.

\section{Results}

By comparing the envelope of the F pulsators to the empirical RRab blue edge on the synthetic HRD we could achieve better agreement, especially at high luminosities. A discrepancy still exists in the low-luminosity regime, perhaps due to the smaller sample of low-luminosity stars (observational bias), and higher uncertainties of the models and in the empirical transformations.

The $\mathrm{F}$ red edge shows a behavior totally different from what the empirical relations suggest. One can see a continuous distribution of stars instead of a sharp red edge. Nonlinear pulsation models give steady $F$ pulsations in this regime. Detailed investigations demonstrated that only very complicated and unlikely evolutionary effects could cause such an absence of pulsators here.

We conclude that it is important that the combined effects of evolution and of mode selection be taken into account in the modeling of RR Lyrae instability strips. Nevertheless a completely satisfactory resolution of the instability strip controversy is still lacking.

Acknowledgments. This work has been supported by Hungarian OTKA (T-026031) and NSF.

\section{References}

Buchler, J.R. \& Kovács, G. 1986, ApJ, 308, 661

Demarque, P., Zinn, R., Lee, Y-W., \& Yi, S. 2000, AJ, 119, 1398

Jurcsik, J. 1998, A\&A, 333, 571

Kovács, G. \& Jurcsik, J. 1996, ApJ, 466, L17

Kovács, G. \& Jurcsik, J. 1997, A\&A, 322, 218

Kolláth, Z., Buchler, J.R., \& Feuchtinger, M. 2000, ApJ, 540, 468

Kolláth, Z. \& Buchler, J.R., 2001, in ApSS Library Series, Vol. 257, Nonlinear Studies of Stellar Pulsations, eds. M. Takeuti \& D. Sasselov, (Kluwer Academic Publishers), 29

Kolláth, Z., Buchler, J.R., Szabó, R., \& Csubry, Z. 2002, A\&A, submitted 